\title{
The Spirit of Bolivia: Complex Behavior Through Minimal Control
}

\author{
Barry Brian Werger ${ }^{1}$ with team members Pablo Funes ${ }^{1}$, \\ Miguel Schneider-Fontán ${ }^{1}$, Randy Sargent ${ }^{2}$, Carl Witty ${ }^{2}$, and Tim Witty ${ }^{2}$ \\ 1 Ullanta Performance Robotics \\ CSCI - SAL 300, 941 West 37th Place (USC), Los Angeles, CA 90089-0781 \\ http://www-robotics.usc.edu/ barry/ullanta \\ 2 Newton Research Laboratories \\ 14813 NE 13th St., Bellevue, WA 98007 - http://www.newtonlabs.com
}

\begin{abstract}
The "Spirit of Bolivia" is a robotic soccer team which demonstrates minimally comprehensive team behavior. By this we mean that each member of the team makes progress towards team goals, and obstructs progress of the opponent, by interacting constructively with teammates and in a sportsmanlike manner with opposing players. This complex behavior is achieved with simple on-board processors running very small behavior-based control programs; team behaviors are achieved withont explicit communication. Externalization - the use of the environment as its own best model - and tolerance - a bias towards reducing the need for accurate information rather than attempting to recognize or correct noisy information - are the keys to robustness and sophistication of team behavior.
\end{abstract}

\section{Introduction}

While it is often tempting to look to more powerful hardware, more complicated programs, more precise sensors, and more communication in the attempt to generate more sophisticated robotic behavior, we feel that the key to success in such an endeavor lies rather in the philosophy of trying to fully exploit minimal systems ([7]). Not only are we far from getting the most out of even the simplest robots; experience has shown that in many cases more precise, "better" robots are more difficult to control ([6]). We present here the "Spirit of Bolivia," a team of RoboCup mid-size soccer-playing robots that exhibit a high degree of sophistication with minimal control and tolerance for - rather than attempts to reduce - imprecision of information. The team is able to keep the ball consistently in play, engaging in constructive offensive and defensive group and individual behavior without explicit communication or modeling of the environment. [1], [3], [2], and [8] have pointed out in various ways the benefits of replacing models of the environment and precise calculation with externalization - the use of the world not only as its own best model, but as a convenient medium for implicit communication.

Though the "Spirit of Bolivia" is primarily a software effort, we next present a brief description of the physical hardware for context. We then describe the 
individual and group behavior of the system, and discuss its performance in the RoboCup ' 97 competition.

\section{The Robots}

The "Spirit of Bolivia" consists of five robots: four fielders (all but one borrowed), Pioneer I robots from ActiveMedia/RWI; and one goalie, built by Newton Labs with an RWI B14 base. A description of the behavior of the goalie appears only here since it is distinct from, and simpler than, that of the fielders.

The financial necessity of borrowing robots for the competition had two major implications: on one hand, the robots were not specialized for RoboCup competition, as were all competing teams; on the other hand, the human team members were able to spend all of their development time on team behavior without uncertainty of hardware reliability or functionality. In hindsight we feel that the tradeoff was highly beneficial.

Robot Vision All of the robots used the Cognachrome [5] vision system from Newton Laboratorie. Based on a $16-\mathrm{MHz} 68332$ microcontroller, the system extracts color-blob information from a video source at a frame rate of $60 \mathrm{~Hz}$. It can be trained to recognize three distinct colors at a time, and outputs the size, visual field location, bounding box, and other data for multiple blobs of each color. It also provides various types of line-tracking data. The robots used wide angle (60-degree) cameras which were not actuated.

The Pioneers - Llaqwa, Sillp'ancho, Salteña, and Ullanta the Robot Levin The Pioneer I robots, as pictured in Figure 1, feature an 18" x14" differentially steered base, five forward-and two side-facing sonars, and grippers (made into large bumpers to comply with RoboCup rules) with contact sensors on each finger. Llaqwa and Ullanta are equipped with the MARS/L system from I.S. Robotics, an on-board 68332 programmed in a Common LISP extended for behavior-based control; Salteña and Sillp'ancho are run with oflboard processors over a radio modem link, programmed in C using the PAI libraries from ActivMedia (Salteña) and P-LOGO (Sillp'ancho).

The only modification made to the Pioneers for RoboCup is the raising of their sonar pingers to a level (two inches higher than normal) where they do not perceive the ball. The pitch-angle of the forward facing camera is set so that a wall at maximum field distance reaches to the top of the field of view, preventing perception of anything outside of the field.

The Goalie - Papa Wayk'u The goalie is an RWI B14 synchronous-steered base with two Cognachrome vision systems for sensing and control. Its simple task is to stay between the ball and the goal while moving along the goal line.

The camera connected to one of the vision systems points out into the field, in order to locate the ball and report its position to the other vision system. To locate the ball, the vision system finds the largest red object below the horizon line; if this is larger than a preset threshold, it is assumed to be the ball. This vision system relays the ball location to the second vision system 60 times per second. 

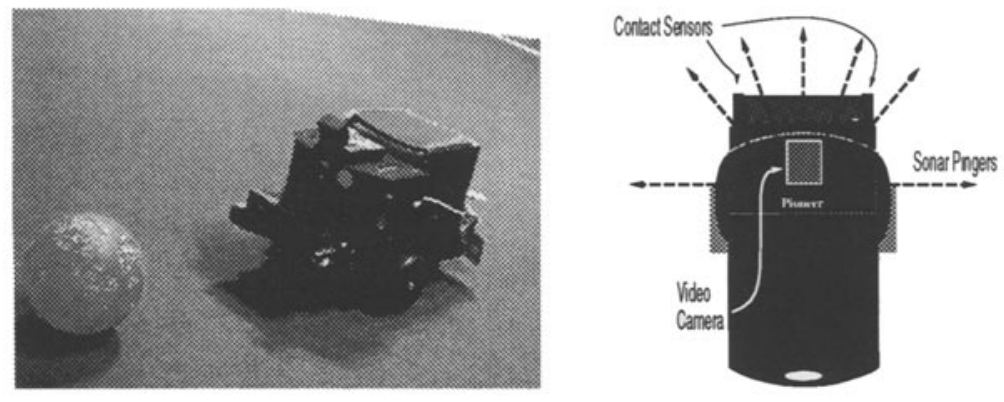

Fig. 1. The Pioneers are differentially steered bases with seven sonar pingers, grippers with contact sensors on the tips, and a Cognachrome vision system.

Since the camera has less than a 180 degree field of view, it can't see the entire field. We point the camera at a 45-degree angle so the goalie looks "ahead and left" (see Figure 2). Whenever the robot doesn't see the ball, it assumes the ball is to its right, and moves to intercept it. By maintaining a "guard" position at the right end of the goal, Papa Wayk'u is able to see the ball whenever it approaches the goal, and quickly zip across to block it or "kick" it out of the way.

The second vision system uses a line-following algorithm to follow along the goal line. It moves left and right based on information received from the first vision system using a simple proportional control loop.

\section{The Behavioral System}

Figure 3 shows the overall structure and information flow of the fielders' behavioral system. There are two main behavioral groupings: the ball-manipulation behaviors centered around the Object Tracker, and the safety-related behaviors Avoidance Generator and Velocity Generator. Team behaviors "emerge" out of the interactions between these two groups.

\subsection{Ball Manipulation}

The fielders manipulate the ball in three ways: pushing it forward, kicking it forward, and batting it to the side. These three forms of manipulation are implemented through a set of basis behaviors [4]; this is a minimal set of behaviors that is sufficient for generating the complex trajectories necessary for soccer play. The set consists of Orbit-CW, Orbit-CCW, and Kick-Ahead, which are illustrated in Figure 4.

These three behaviors are mutually inhibitory in such a way that only one is active at a time. Changes of active status happen at a potential rate of $10 \mathrm{~Hz}$, 


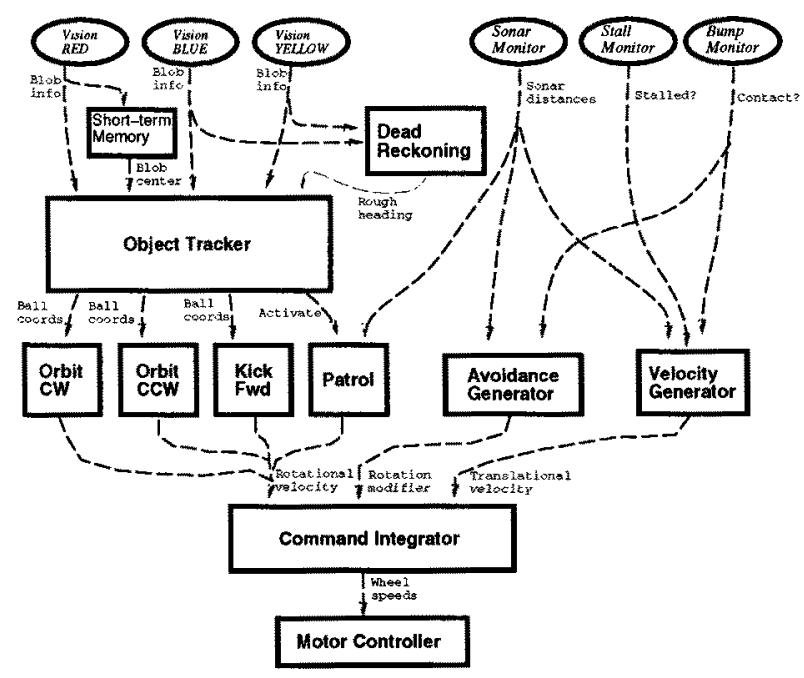

Fig. 3. The control system of the fielders. Boxes are individual behaviors; ovals are perceptual devices; and links are labeled with the type of information they carry.

degrees of "north", 180-degree distinction of east and west) and a rough calibration of the dead-reckoning whenever either of the goals is seen with some degree of confidence results in robust orientation.

Behavior Tables and Short-Term Memory The basis behaviors are implemented as simple tables which divide the field of view into a $5 \times 5$ grid; each section of the field of view has an associated rotational velocity (Figure 4). When the ball leaves the field of view, the short-term memory (Figure 3) feeds the most-recently-viewed ball position into the Object Tracker for a period of a few seconds. With this in mind, we have built "recovery behaviors" into the edges of the behavior tables. When the ball
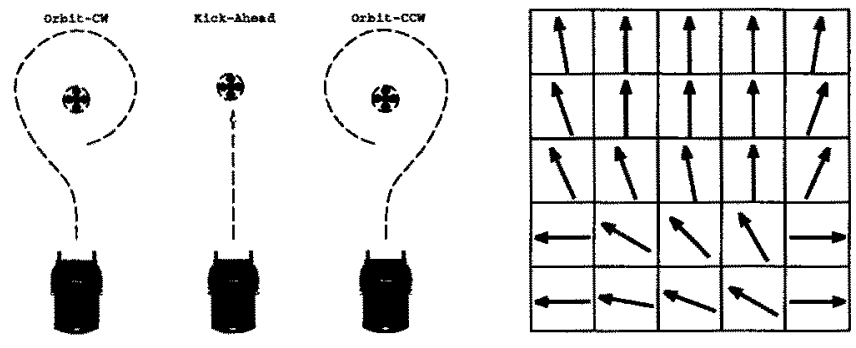

Fig. 4. The three basis behaviors sufficient to generate ball-manipulation for soccer play. Each is implemented as a table that assigns rotational velocities to segments of the visual field, such as the one shown for Orbit-CW. 


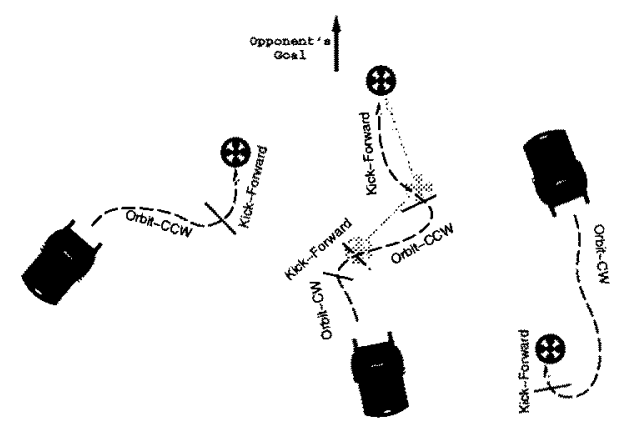

Fig. 5. Some typical trajectories generated by combination of the ball-manipulation basis behaviors.

does leave the robot's field of view, the robot takes an action which is very likely to cause it to regain direct sight of the ball. This allows the robots to react to an unseen ball without explicitly calculating motion of the ball within some model of the environment, though in practice it appears that the robot is doing just that. The same effect is what allows the Orbits to function. As can best be understood by imagining the ball at various positions in the table in (Figure 4), the robot actually "tacks" around the ball as it loses and regains sight of it. This tacking behavior also causes it to contimuously change its angle so that it is very likely to line up for a switch to $K i c k$-Ahead behavior during the interpolation process.

Pushing, Batting, and Kicking Pushing is the simplest of the manipulations; when the ball is directly in front of the robot, the Kick-Ahead behavior moves the robot forward as fast as possible, pushing the ball and making small corrections until such time that the perceptual situation and Object Tracker determine that major (Orbitbased) corrections are necessary. Batting occurs when the ball is stuck against a wall or being advanced by an opponent; the combination of tacking behavior inherent in interpolation and reduced speed due to the Velocity Generator's perception of the wall or opponent causes the robot to swing back and forth more forcefully as it gets closer to the ball, always remaining on the proper side of the ball for interpolation. This results in the ball being batted (by the side of the gripper) towards the opponent's goal. Kicking results from the observation that in certain configurations, there is no way that the robot can push or bat the ball productively without backing up (which would involve complex, sequenced behavior). This occurs when the ball is right next to the front of the robot (at the lower corners of the visual field) where forward motion wonld at best knock it to the side (Figure 6). When the ball is in such a position, the robot rotates at high speed away from it, loses sight of it, and thus continues rotating (due to short term memory) for a full rotation. Since the robot is oblong, its rear-end often "kicks" the ball smartly forward.

\subsection{Safety Behaviors and Motor Command Integration}

The Velocity Generator outputs a speed inversely proportional to the distance to the closest object detected by the sonar; the Avoidance Generator outputs rotational ve- 


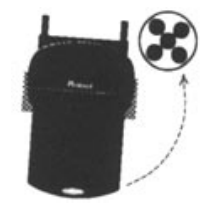

Fig. 6. The proper configuration for a rear-end kick.

locities that cause the robot to turns away from objects to its sides. The Command Integrator combines the rotational velocities from the ball-manipulation behaviors and the translational velocity from the Velocity Generator to form final wheel-speeds. The Avoidance rotations override the manipulation rotations only when necessary. The Velocity Generator monitors stalls and gripper contact points to detect situations where the sonars do not properly register collisions.

\section{Team Behavior}

Team behavior arises from the interaction of obstacle avoidance, ball manipulation, and short-term memory. In a manner akin to the Dispersion basis behavior used in [4], fielders move away from objects close to their sides. This dispersion leads to effective offensive and defensive formations of some sub-set of the team when combined with ball manipulation behaviors. When advancing towards the opponent's goal, team members fall into the configuration seen in Figure 7: one robot leads with the ball, while others stay to the side and behind, ready to recover the ball should it be fumbled or stolen. The robots on the sides stay slightly behind because they slow down as they draw abreast of the ball and turn more sharply towards it, perceiving the leader with sonar. It is not uncommon that possession of the ball changes between members of the advancing group as it moves. The number of robots participating in the group is limited by perception; after some critical mass (three, in practice), robots on the edge of the group tend to lose perception of the ball for longer than the short-term memory duration and take up a Patrol behavior.
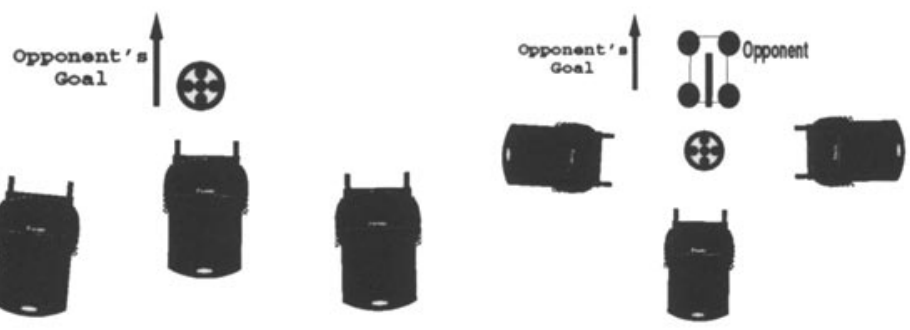

Fig. 7. Offensive (left) and defensive (right) group formations. 
In a defensive situation the ball is not advancing, so the robots fall into a semicircular arrangement around it. This effectively prevents the opponent from dodging the defense, and places players in a good position to gain possession of the ball. The offensive or defensive response is triggered solely by the behavior of other agents and the ball, with no need for symbolic distinction of teammates from opponents.

Given the lack of information about opponent strategies, we felt that the benefits of homogenizing the fielders outweighed those of specializing them for field positions. To prevent clustering, however, it proves effective to vary the direction of rotation of the Patrol behavior, which is implemented quite simply as a fixed rotational velocity. Robots with different directions of rotation tend to wander separate "territories, due to effects of safety behaviors during interactions.

\section{Summary of Control System}

The "Spirit of Bolivia" demonstrates an outstanding level of behavioral sophistication in the RoboCup mid-size field, including polite "sportsmanlike" behavior towards opponents, constructive interaction with teammates, and ability to continuously manipulate the ball both offensively and defensively. This is all done with almost no internal state (only the dead-reckoning position used when neither goal can be perceived, and the short-term memory), no explicit communication, and with extremely simple code: the most complex behavior is the Object Tracker presented in pseudocode in Section 3.1, and the entire system occupies fewer than four pages of LISP code. The system has redundancies (e.g., dead-reckoning), seamless recovery procedures (e.g., edges of basis behavior grids), and a minimal need for accurate information that all lead to extremely robust, purposeful, and lively behavior.

\section{Performance at RoboCup '97}

Though not one of the co-champions, the "Spirit of Bolivia" was the only team at RoboCup whose defense proved impenetrable (no points were scored against the team). The team did not have a chance to play against either of the championship teams during the competition, but in an unofficial game shortly after the finals, the "Spirit of Bolivia" won against one of them, ending up as the only team to have won against either of the co-champions, who consistently tied against each other. Given that our team was the only one attempting obstacle avoidance, which had a major impact on the robots' speed and ability to maneuver, we consider the "Spirit of Bolivia" to be a great success.

\section{Acknowledgements}

We would like to thank all the people who were so generous in lending us their precious robots, including everyone at ActivMedia, RWI, and Newton Labs, the organizers of RoboCup for an excellent - and difficult! - job, and all those intrepid souls who dealt with the daunting task of getting the robots to Japan and back, especially Merri Naka, Jon Reese, and Anne Wright for the slick airline cargo idea... 


\section{References}

1. Beckers, R., O. Holland, and J. Deneubourg. From Local Actions to Global Tasks: Stigmergy and Collective Robotics. Proceedings of the 4th International Workshop on the Synthesis and Simulation of Living Systems, 1994.

2. Brooks, R. Intelligence Without Reason. Computers and Thought - IJCAI 91.

3. Donald, B., J. Jennings and D. Rus: Information Invariants for Distributed Manipulation. International Journal of Robotics Research, 16(5), 1995.

4. Mataric, M.: Issues and Approaches in the Design of Collective Autonomous Agents. Robotics and Autonomous Systems 16, 1995.

5. Sargent, R., B. Bailey, C. Witty, and A. Wright: Dynamic Object Capture Using Fast Vision Tracking. AI Magazine 18(1), 1997.

6. Smithers, T.: On Why Better Robots Make it Harder. Proceedings of SAB-94.

7. Werger, B.: Principles of Minimal Control for Comprehensive Team Behavior. Ullanta Performance Robotics Technical Report \#97-02

8. Werger, B. and M. Mataric. Robotic Food Chains: Externalization of State and Program for Minimal-Agent Foraging. Proceedings of the 4th International Conference on Simulation of Adaptive Behavior (SAB '96). 